\title{
Bibliotheksbau
}

Martin Nissen*

\section{Die Sanierung des Südflügels der Universitätsbibliothek Heidelberg (2016-2019)}

https://doi.org/10.1515/bfp-2020-2022

Zusammenfassung: Im Mai 2019 wurden das Informationszentrum sowie das Multimediazentrum im Südflügel des Altbaus der Universitätsbibliothek Heidelberg nach rund zweieinhalbjähriger Bauzeit wiedereröffnet. Durch Entfernung von in den 1980er-Jahren eingezogenen Stahlgalerien entstanden zwei Großräume, die auf Grundlage eines zonierten Konzeptes verschiedene Nutzungen zusammenbinden. Der Beitrag beleuchtet die Vorgeschichte sowie die Durchführung der Baumaßnahme und bewertet das Nutzungskonzepts nach rund einjähriger Betriebszeit.

Schlagwörter: Universitätsbibliothek Heidelberg; Bibliotheksbau; Bibliotheksnutzung

\section{The Renovation of the South Wing of Heidelberg University Library (2016-2019)}

\begin{abstract}
In May 2019, the Information Centre and the Multimedia Centre in the south wing of the old building of Heidelberg University Library were reopened after a construction period of about two and a half years. By removing the steel galleries, which had been built in the 1980s, two large rooms were created that combine different uses. The article sheds light on the prehistory as well as on the construction measure and evaluates the concept after about one year of operation.
\end{abstract}

Keywords: Heidelberg University Library; library building; library use

\section{Einleitung}

Nach rund 30 Jahren ohne größere Umbau- oder Sanierungsmaßnahmen wurde ab 2008 damit begonnen, die bauliche Situation des Hauptgebäudes der Universitätsbibliothek Heidelberg in der Heidelberger Altstadt grund-

*Kontaktperson: Dr. Martin Nissen, Nissen@ub.uni-heidelberg.de legend $\mathrm{zu}$ ertüchtigen und $\mathrm{zu}$ erweitern. ${ }^{1}$ Die mit rund 21 Millionen Euro und einer Nutzfläche von 7100 Quadratmetern umfangreichste Maßnahme war die Norderweiterung der Universitätsbibliothek mit der Sanierung der oberen vier Geschosse des angrenzenden Triplexgebäudes, die seitdem als Lesesaal mit rund 1000 Arbeitsplätzen und Büroflächen für rund 80 Mitarbeiter genutzt wird. ${ }^{2}$ Hinzu kam 2009-2015 die Neugestaltung eines Handschriftenlesesaals im Ostflügel des Altbaus, die Sanierung der Ausleihe im Westflügel, die Generalüberholung von Geschossflügeln der Freihandmagazine sowie diverse kleinere Maßnahmen, die insbesondere die brandschutztechnische Ertüchtigung der Treppenhäuser und Aufzüge umfasste und die teilweise noch nicht abgeschlossen sind (Bauabschnitte UB-Altbau I-III). Der neu geschaffene Lesesaal im Triplex-Gebäude der 1970er-Jahre zeichnet sich durch seine modern-funktionale Architektur mit einer großzügig bemessenen Lese- und Lernlandschaft aus. Im Altbau von 1905 wurde bei den Sanierungsmaßnahmen behutsam eingegriffen, um, wo immer möglich, Mobiliar und bauliche Elemente aus der Entstehungszeit zu bewahren bzw. dem ursprünglichen Charakter entsprechend neu zu gestalten.

Durch die Erschließung des Triplex-Gebäudes sowie die Sanierungsmaßnahmen im West- und Ostflügel des Altbaus wurde der Sanierungsrückstau im zentral gelegenen Südflügel des Altbaus augenscheinlich. Der Südflügel mit seiner prächtigen Sandsteinfassade bildet das Zentrum der vierflügeligen schlossartigen Anlage. Im nördlichen Teil dieses Südflügels waren nach dem Abriss des Historischen Lesesaals in den Jahren 1953-1956 zwei Säle entstanden, die seitdem verschiedene Nutzungen erfuhren und in den 1980er-Jahren durch den Einzug von Stahlgalerien weiter verdichtet wurden. Die Norderweiterung im Triplex-Gebäude, die neben der Aufnahme von 1000 Arbeitsplätzen auch Platz für die Aufstellung von rund 200000 Bänden bietet, machte den Weg frei, den Altbau durch Entfernung der Galerien wieder aufzulockern und die beiden großen Funktionsräume sowie das darunterliegende Kellergewöl-

1 Vgl. dazu zuletzt: Probst und Balzuweit (2020).

2 Vgl. ausführlicher: Balzuweit und Nissen (2016). 
be in einer weiteren Baumaßnahme zwischen 2016-2019 grundlegend $\mathrm{zu}$ sanieren und damit energetisch und brandschutztechnisch auf den neuesten Stand zu bringen.

Im Folgenden werden die Vorgeschichte, die Ziele und die Durchführung der Generalsanierung von Informationszentrum und Multimediazentrum im Südflügel - in der internen Zählung Bauabschnitt IV UB-Altbau - dargestellt und eingeordnet. Die Gesamtbaukosten lagen bei 6 Millionen Euro, so dass sich für die Altbausanierung in den ersten vier Bauabschnitten eine Gesamtsumme von 14 Millionen Euro ergibt, die das Land Baden-Württemberg investierte.

\section{Der Südflügel 1905-2015}

Der nach der Konzeption des Heidelberger Oberbibliothekars Karl Zangemeister (1837-1902) entworfene und nach Plänen des badischen Architekten und Baumeisters Josef Durm (1837-1919) gestaltete Altbau wurde im Jahr 1905 eröffnet. Zentraler Raum des Gebäudes war der gegenüber dem Haupteingang im Erdgeschoss gelegene Lesesaal, der mit einer Grundfläche von 320 Quadratmetern und einer die übrigen Geschosse überragenden Raumhöhe von etwa 10 Metern als Kathedrale der Wissenschaft gestaltet war. Der Aufsicht führende Bibliothekar überwachte den Saal von einem Arbeitsplatz aus, vis-à-vis dem Eingang gelegen (auf der Abb. 1 auf der linken Seite).

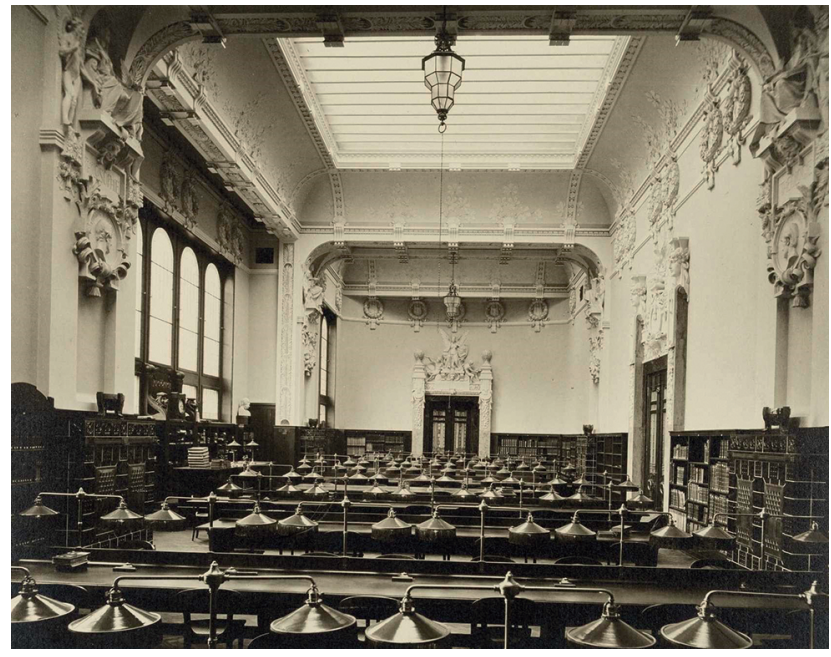

Abb. 1: Historischer Lesesaal (1905), (Foto Gottmann) ${ }^{3}$

Während der Altbau auf eine Universitätsgröße von ca. 1500 Studierenden $^{4}$ ausgelegt war, konnte das Gebäude

3 https://heidicon.ub.uni-heidelberg.de/detail/548383.

4 Vgl. Rosenbusch (1900) 22. spätestens seit den 1950er-Jahren den wachsenden Anforderungen nicht mehr gerecht werden. Hinzu kam der anti-historistische Impetus der Nachkriegszeit, der in einem funktional-versachlichten Baustil eine Abwendung von der Vergangenheit suchte. Der ursprüngliche Lesesaal wurde 1953 unter der Regie des Staatlichen Hochbauamts abgebrochen..$^{5}$ Auf der Grundfläche entstand bis 1956 ein doppelgeschossiger Baukörper mit zwei Großräumen à 320 Quadratmetern. Der untere Saal nahm den Katalog der Universitätsbibliothek, Nachschlagewerke sowie die bibliografische Auskunft auf. Der obere Saal diente als Lesesaal mit 120 Arbeitsplätzen und einer Handbibliothek von 7500 Bänden. Abgeschlossen wurde der Baukörper durch eine flache Lichtdecke mit einem Pultdach aus Blechplatten mit drei aufgesetzten Glashäusern, dessen bauliche Mängel in den folgenden Jahrzehnten immer wieder Probleme verursachten und nicht nur zu Wassereinbrüchen, sondern sogar zum Herabstürzen von Deckenelementen führten.

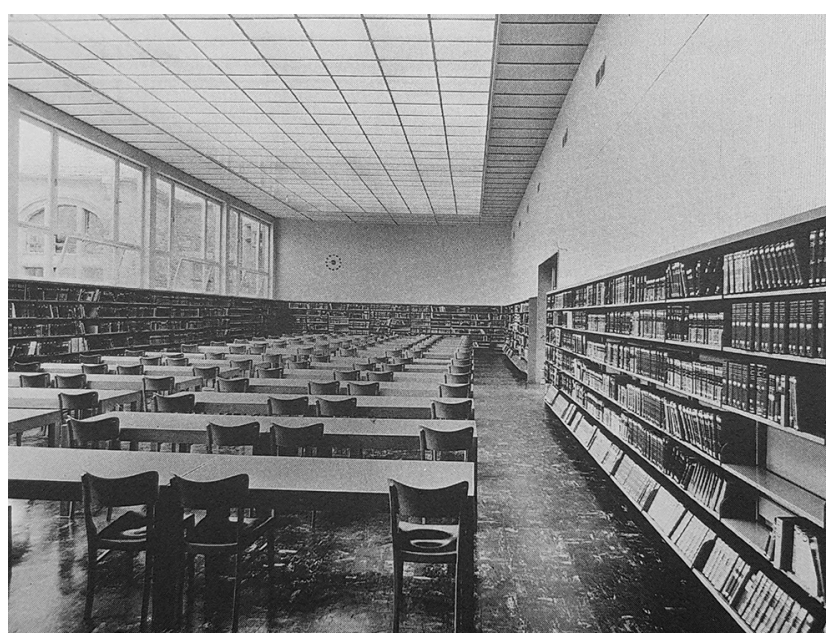

Abb. 2: Lesesaal (1955) nach dem Abbruch des alten Lesesaals (Foto Lossen) ${ }^{6}$

Zwischen 1985 und 1987 wurden in beiden Sälen Stahlgalerien eingezogen. Dazu war aus statischen Gründen das Einbringen weiterer Stahlträger, die bis in das Sockelgeschoss reichten, erforderlich. Diese Maßnahme war Teil umfangreicher Sanierungsarbeiten (1979-1987), mit denen man die wichtigsten baulichen Mängel beheben und behelfsmäßig auf den gesteigerten Platzbedarf reagieren wollte. ${ }^{7}$ Der Lesesaal wurde in die beiden oberen Ge-

\footnotetext{
5 Ewald (1989) $33 \mathrm{f}$.

6 Mittler (1989) 17.

7 Ewald (1989) $78 \mathrm{ff}$.
} 
schosse des Magazintrakts verlegt. Die drei Stockwerke im Südflügel inklusive der Galerien wiederum wurden bis 2015 mehrfach an die sich ändernden Gegebenheiten und Erfordernisse angepasst. Im Erdgeschoss waren bis 2003 die Katalogauskunft, die alphabetischen und systematischen Zettelkataloge, die Kataloge der Sonderbestände der UB sowie Katalogrecherche-PCs für den ersten elektronischen Bibliothekskatalog angesiedelt. Im Obergeschoss befanden sich zunächst die bibliografische Auskunft, die Fernleihannahme, die überregionalen Kataloge und die Fachbibliografien. Im Gewölbe im Untergeschoss wurde der Gesamtkatalog der Institutsbestände, der vormals an wechselnden Standorten in der Altstadt angesiedelt war, aufgestellt, bis dort 1995 der erste größere CIP-Pool mit 30 teilweise multimedialen PC-Arbeitsplätzen eingerichtet wurde, ergänzt um den Bereich AV-Medien mit 10 Videound Ton- sowie 6 Mikroformarbeitsplätzen. Der Zuwachs an elektronischen Datenbanken und retrospektiv katalogisierten Titelaufnahmen sowie die nachlassende AV-Medien-Nutzung ermöglichten in der Folge die Aufstellung dringend benötigter PC-Nutzerplätze. Ab 2004 wurden im Untergeschoss die Fachbibliografien aufgestellt und ein erster PC-Schulungsraum eingerichtet. Im Erdgeschoss wurden Katalog- und bibliografische Auskunft zusammengeführt und im Obergeschoss auf den frei gewordenen größeren Flächen ein neues Multimediazentrum gegründet. In den folgenden Jahren konnte die Anzahl der gedruckten Bibliothekskataloge, allgemeinen Nachschlagewerke und Fachbibliografien weiter reduziert werden, so dass das Untergeschoss freigestellt und dort ab 2007 ein ohne Bedingungen frei zugänglicher sogenannter „Laptoplernraum“ eingerichtet werden konnte, der rasch eine hohe Nutzung erfuhr.

Die Stahlgalerien, die die beiden Großräume zerschnitten, waren dabei immer nur als Kompromiss zu verstehen und von Beginn an auf einen möglichen Rückbau bei verbessertem Raumangebot hin konzipiert. Der Architekt Hans Peter Stichs vollzog den Spagat zwischen der Sanierung des historischen Treppenhauses im Südteil des Südflügels durch Wiederherstellung der in den 1950er-Jahren abgerissenen Glastonne einerseits und dem Einzug dieser von Beginn an bei der Direktion, den Architekten und Nutzern unbeliebten Galerien andererseits. Nach Abschluss der Sanierungsarbeiten 1989 äußerte er sich wie folgt:

„Die Möglichkeit, auf Dauer Durms Gesamtkonzeption wieder realisieren zu können, war eines unserer Hauptanliegen. Wir wollten durch die Art unserer Um- und Erweiterungsbauten erreichen, daß an dem Tag, an dem durch die endgültige Umstellung der gesamten Kataloge auf elektronische Medien jeder Benutzer in der Lage ist, über sein persönliches Bildschirmgerät jede Information, jede Textseite sich ausdrucken zu lassen, daß an diesem Tag die 50er-Jahre-Betonkonstruktion und unsere neuen Stahlgalerien abgerissen werden und daß unter unserem neuen Dachgeschoss der alte Lesesaal als Lesesaal original mit allen Innenraumstukkaturen, den alten Decken, Leuchten, Farben, Boden, Holzeinbauten, der Sandsteinfassade außen wieder eingebaut wird - als geistiger Mittelpunkt der Universität! Möglich ist es - wir hoffen, bald.“"

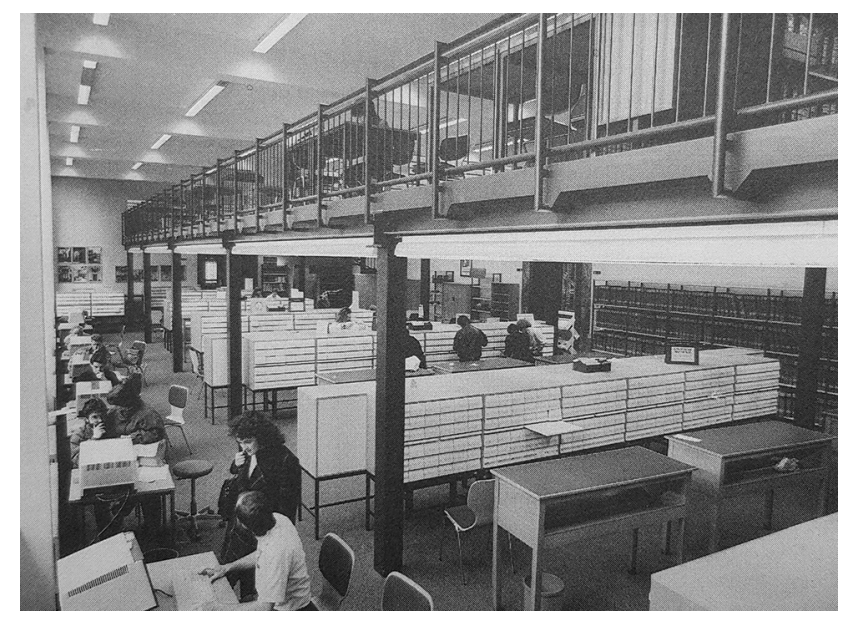

Abb. 3: Informationszentrum 1988 (Foto Kresin) ${ }^{9}$

Die baulichen Mängel im Informationszentrum (IZA) und Multimediazentrum (MMZ) wurden spätestens seit den 2000er-Jahren offenbar. Unzulängliche Anschlüsse führten wiederholt zu Wasserschäden im Deckenbereich, die Fenster waren nicht nur durch das Eintreten von Feuchtigkeit zwischen den Scheiben teilweise blind, sondern führten auch durch beschädigte Schließmechanismen zu einer unzulänglichen energetischen Situation. Der Putz an den Wänden war in Teilen schadhaft, die Teppichböden durch Jahrzehnte lange intensive Nutzung abgetreten. Hinzu kam, dass die eingezogenen Galerien nicht nur den Raum zerschnitten und den Raumeindruck störten, sondern dass die dortigen Arbeitsplätze aufgrund verschärfter brandschutztechnischer Vorgaben und fehlender Fluchtwege seit 2015 nicht mehr genutzt werden konnten.

Ursprüngliche Überlegungen, den alten Lesesaal wiederherzustellen, wurden aufgrund des erforderlichen Platzbedarfs verworfen. Auf die Verdichtung der 1950erund 1980er-Jahre erfolgte durch den Ausbau der Galerien eine Entflechtung des Gebäudes. Voraussetzung dafür waren die freiwerdenden Flächen im Triplex-Lesesaal, die einen Teil der bibliografischen Hilfsmittel, die bis dahin auf der Galerie des unteren Großraums standen, aufnah-

8 Stichs (1989) 130. 9 Mittler (1989) 15. 


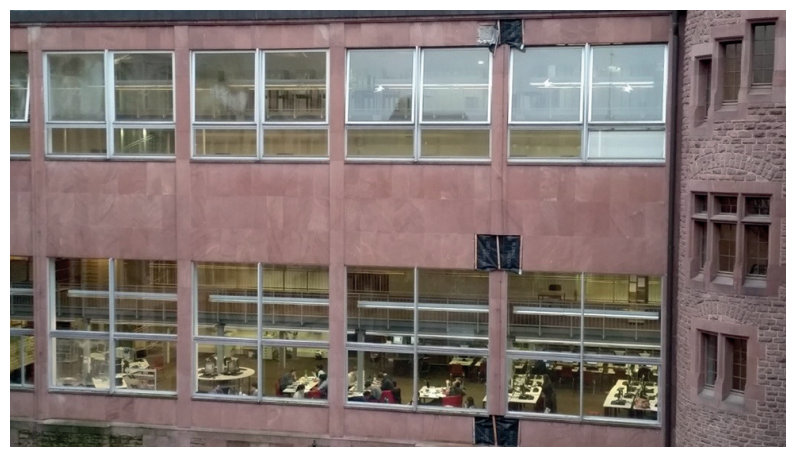

Abb. 4: Nordfassade des Südflügels (2017), mit abgenommenen Sandsteinplatten (Foto: Martin Nissen)

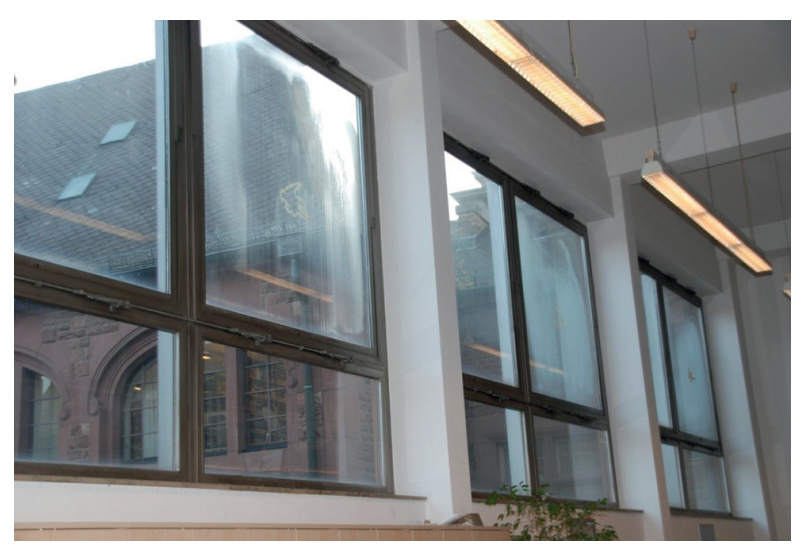

Abb. 5: Multimediazentrum (2014), Fensterfront mit blinden Fenstern (Foto: Martin Nissen)

men. Weitere Bibliografien wurden aufgelöst bzw. durch elektronische Datenbanken ersetzt. Die Mikroformensammlung wurde ins Tiefenmagazin verlagert, die AVSammlung, die überwiegend aus Fernsehmitschnitten bestand, zum größten Teil aufgelöst.

\section{Ziele und Durchführung der Baumaßnahme}

Nach dem Einbau eines neuen Treppenhauses (inkl. des Einbaus neuer Toilettenanlagen im Untergeschoss), der Sanierung der Ausleihe (inkl. der Sanierung eines Magazingeschosses) und der Sanierung vier weiterer Magazingeschosse (inkl. des Lesesaalzugangs) war die Sanierung der Nordseite des Südflügels der vierte Bauabschnitt bei der Sanierung des Altbaus (BA IV). Auch wenn die Idee, den ursprünglichen Lesesaal wiederherzustellen, verworfen werden musste, entwickelten die Architekten von ap88 das Konzept der „typologischen Rekonstruktion“, bei dem die Konstruktion auf das ursprüngliche Traggerüst (der 1950erJahre) reduziert wurde und die Wiederherstellung in Anlehnung an den ursprünglichen Bau (von 1905) erfolgte.
Inklusive vorbereitender Maßnahmen erfolgte die Sanierung zwischen September 2016 und April 2019. Der Zeitplan konnte mit einer Bauzeit von zweieinhalb Jahren eingehalten werden. Die Gesamtbaukosten beliefen sich auf sechs Millionen Euro. Die feierliche Eröffnung fand am 2. Mai 2019 statt.

Die Baumaßnahme verfolgte drei Ziele. Im Mittelpunkt stand zunächst die Behebung baulicher, insbesondere brandschutztechnischer Mängel. Hierzu diente u.a. der Ausbau der Galerien ab August 2017. Zentral war ferner die energetische Sanierung, die insbesondere durch die komplette Neugestaltung der Nordfassade sowie den Einbau einer Belüftungsanlage erreicht wurde. Zuletzt ging es darum, Funktionalität und Aufenthaltsqualität der Räumlichkeiten zu verbessern. Die Bauleitung lag wie bei der Sanierung des Triplex-Lesesaals bei Vermögen und Bau Baden-Württemberg - Amt Mannheim und Heidelberg, das erneut die Heidelberger Architektenpartnerschaft ap88 mit der Ausführung betraute.

Nachdem die Räume zur Vorbereitung der Sanierung im März 2017 geschlossen worden waren, wich das Multimediazentrum ins 5. Magazingeschoss West aus, das mit rund 200 Quadratmetern Nutzfläche einen fast vollwertigen Ersatz für den zentralen PC-Pool der Universität in der Altstadt bot. Beim Informationszentrum (IZA) waren die Einschränkungen in der Interimszeit gravierender. Durch Umzug in ein Großraumbüro im Erdgeschoss des Südflügels musste die Zahl der Recherche-PCs stark reduziert werden, was durch das Aufstellen weiterer Recherche-PCs im Zugangsbereich zum Lesesaal in gewissem Umfang kompensiert wurde. Die bibliografische Auskunft fand auch während der Bauphase ohne Einschränkungen statt.

Wie bei vorherigen Baumaßnahmen wurde auf längere Schließungsphasen verzichtet. Das Multimediazentrum wurde bei Auszug und Einzug je für zwei Tage geschlossen. Auszug und Wiedereröffnung des Informationszentrums erfolgten im laufenden Betrieb.

Äußerlich sichtbarster Ausdruck der Sanierungsmaßnahme war die Neugestaltung der zum Innenhof gelegenen Nordfassade des Südflügels. Im Grundsatz wurde die Gliederung durch schmale Streben der Fassade der 1950erJahre aufgegriffen. Die Zahl der Streben wurde erhöht, um die vertikale Gliederung der Fensterflächen zu betonen. Zudem wurden die horizontalen Gliederungselemente aufgegeben und die Fenster bodentief gestaltet, wodurch die Fassade als bindendes Glied die Funktion einer Klammer für beide Geschosse übernahm. Die Streben wurden wie bei dem Vorgängerbau in Sandstein gefasst. Auch hierdurch fügt sich die Fassade in den historischen Innenhof des Magazintrakts ein. Verstärkt wird dieser Eindruck durch eine zurückhaltende Gestaltung der oberen Ab- 
schlussplatten durch mit CNC-Technik in Sandstein gefräste florale Ornamente, die eine Reminiszenz an die prächtig geschmückte Südfassade sowie weitere Schmuckelemente an der West- und Ostfassade darstellt. Die Belüftung der Räume wird - nach Abschluss der Sanierung des Dachgeschosses des Altbaus - durch eine Belüftungsanlage erfolgen, die unter dem Dach des Südflügels eingebaut werden muss. Bis dahin erfolgt die Belüftung konventionell über die manuell bedienbaren RDA-Klappen.

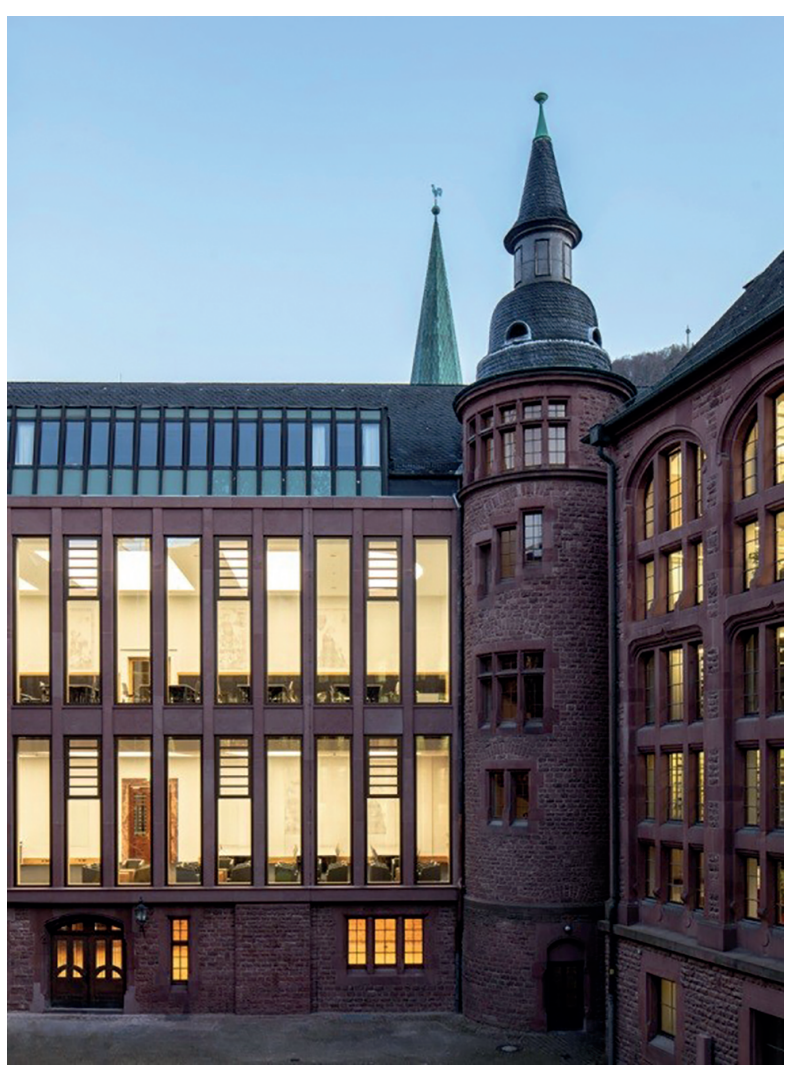

Abb. 6: Neu gestaltete Nordfassade des Südflügels 2019 (Foto: Altenkirch)

Der Innenraum des Informationszentrums vermittelt einen erhabenen Raumeindruck. Durch die Entfernung der Galerie ist die Raumhöhe von 6 Metern wieder sichtbar. Die Offenheit des Raumes wird durch eine die Symmetrie der Fassadenstreben aufgreifende Lichtdecke verstärkt. Ein Gegengewicht zu der symmetrischen Strenge von Nordfassade, Lichtdecke und Südwand bilden die geschwungenen Stirnseiten, die mit modern interpretierten raumhohen Regalwänden in Eiche verkleidet sind. In diese eingestellt ist der historische Bandkatalog, der in 734 Bänden den Bestand bis 1935 verzeichnet. Da die darin eingefügten Katalogkarten vollständig über den digitalisierten Katalog Digikat recherchierbar sind und alle in neuerer Zeit einmal entliehenen Werke zudem über den Online-Katalog HEIDI ermittelbar sind, hat der alphabetische Bandkatalog seine Funktion verloren. Die Benutzung ist lediglich in seltenen Fällen für das Nachschlagen von Provenienzen, die teilweise auf der Rückseite der Katalogkarten verzeichnet sind, erforderlich. Die Aufstellung des Bandkatalogs an so prominentem Ort ist eine Reminiszenz an die langjährige Nutzung dieses Raums als Katalogsaal, repräsentiert er doch den durch die Heidelberger Bibliothekare geschaffenen Zugang zu den Sammlungen der Universitätsbibliothek.

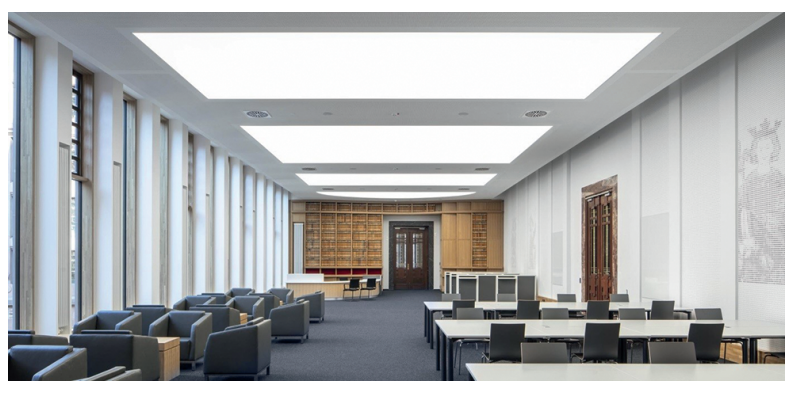

Abb. 7: Informationszentrum Altstadt 2019 (Foto: Altenkirch)

Einen weiteren Bezug zum historischen Bestand bilden die vier Motive aus dem Codex Manesse, die mittels Lochtechnik in die Verkleidung der Südwand eingebracht wurden. ${ }^{10}$ Diese verbessern neben den Regalwänden, dem Teppichboden und der abgehängten Lichtdecke die akustische Situation. Im Multimediazentrum im Obergeschoss wurden die vier Motive als Ausschnitte wiederaufgenommen. Unterhalb der Akustikverkleidung dient die umlaufende Verkleidung aus anthrazitfarbenen mitteldichten Holzfaserplatten (MDF) mit schmalem längsverlaufendem Rillenprofil als zusätzliches Schallschutzelement.

Im Vergleich zum Informationszentrum ist das Multimediazentrum der Funktion des Raumes entsprechend moderner gestaltet. Auch hier geben die raumhohen Eichenfenster den Blick nach Norden in den historischen Innenhof frei. Der ruhige Raumeindruck wird durch die Symmetrie von Fassadengliederung, umlaufender Verkleidung, Lichtdecke sowie das spiegelbildlich aufgestellte Mobiliar verstärkt. Die Auskunftstheke ist seitlich positioniert und tritt, da die Beratung überwiegend an den Computerarbeitsplätzen bzw. Scanstationen stattfindet, stärker in den Hintergrund. Die umlaufende Verkleidung dient der Aufnahme sowohl von technischen Vorrichtungen als auch von Stauraum, so dass der aufgeräumte Raumeindruck keine Störung erfährt.

10 Die vier ausgewählten Motive sind: Kaiser Heinrich VI., CPG 848, 6r, König Konradin der Junge, CPG 848, 7r (rechte Figur), König Wenzel II. von Böhmen, CPG 848, 10r, Herr Walther von der Vogelweide, CPG 848, $124 \mathrm{r}$. 


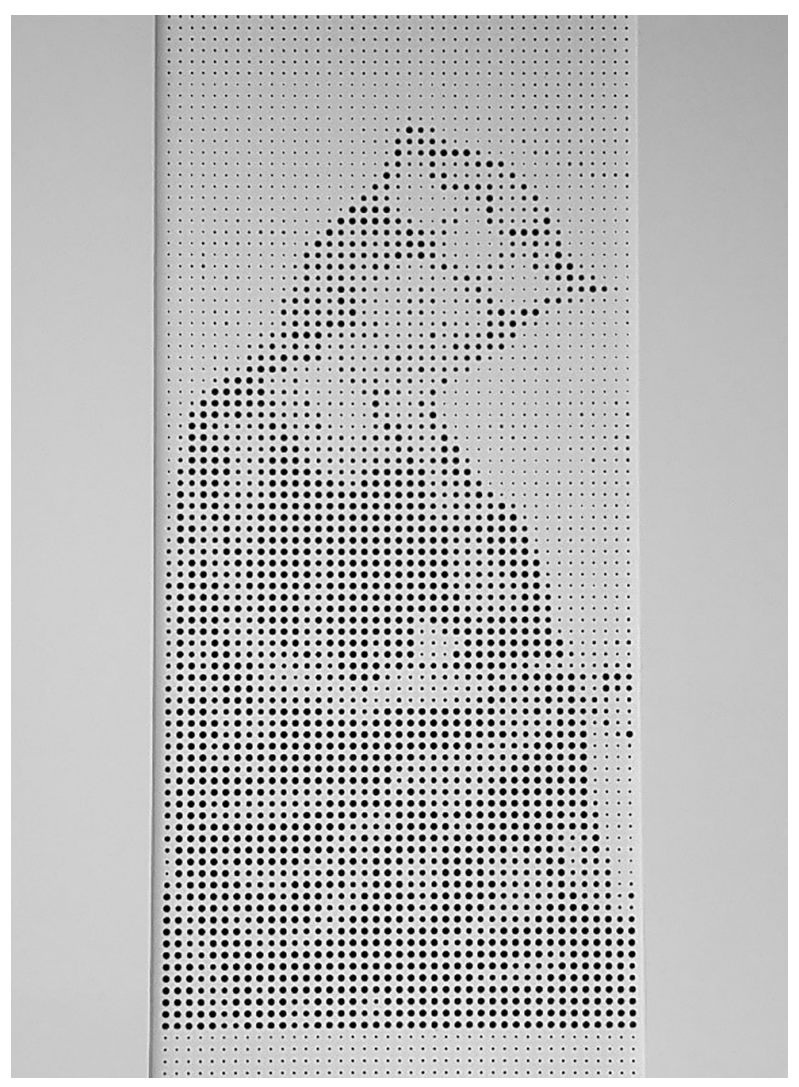

Abb. 8: Lochbild. Motiv aus dem Codex Manesse (CPG 848), Herr Walther von der Vogelweide, $124 \mathrm{r}$ (Foto: Martin Nissen)

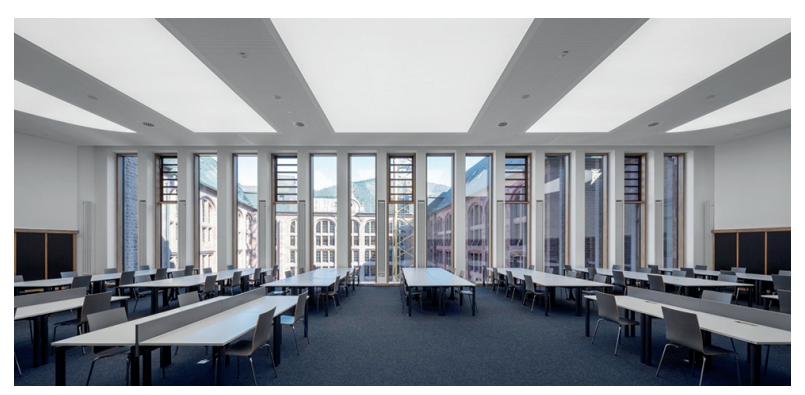

Abb. 9: Multimediazentrum 2019 (Foto: Altenkirch)

\section{Nutzungskonzept}

Das Nutzungskonzept schließt - unter verbesserten räumlichen Bedingungen - an das vorher bestehende Nutzungskonzept an. Nach Abschluss der Sanierung wurde im Obergeschoss erneut das Multimediazentrum mit dem zentralen PC-Pool für Mitglieder der Universität in der Altstadt eingerichtet. Die 56 fest installierten Computerarbeitsplätze werden ergänzt durch 27 frei zu nutzende Laptoparbeitsplätze sowie sechs Sondernutzungsplätze, die auch externen Nutzerinnen und Nutzern offenstehen. Neben einem DVD/VHS-Abspielgerät, verschiedenen Scanstationen zur Herstellung digitaler Kopien, auch von Diapositiven und Schallplatten, sind hier die Universal-Readerprinter zur Nutzung der Mikroformensammlung zu nennen. Diese bieten die Möglichkeit, automatisiert digitale Kopien von Mikrofilmen und Mikrofiches zu erstellen.

Während im Multimediazentrum (MMZ) die Nutzung durch Universitätsmitglieder, insbesondere Studierende, im Mittelpunkt steht, ist das Informationszentrum im Erdgeschoss die erste Anlaufstelle für alle Besucher. Die 36 Recherche-PCs mit Internetzugang und Zugriff auf den Großteil der lizenzierten Ressourcen werden allen Nutzerinnen und Nutzern angeboten. Die Erstinformation als zentrale Funktion des Raumes wird durch eine zentral gelegene, vom Eingang aus gut sichtbare Auskunftstheke mit vier Arbeitsplätzen für die Kolleginnen und Kollegen der Benutzungsdienste und die Auskunft des Rechenzentrums deutlich gemacht. Ein Arbeitsplatz verfügt dabei über eine höhenverstellbare Theke, die als Sitz- oder Steharbeitsplatz genutzt werden kann. Hinzu kommen im Informationszentrum zwei Scanstationen mit Druck-, Kopierund Scanfunktion sowie eine Leselounge mit 20 Lesesesseln und fünf Stromladewürfeln, die sich zum Innenhof hin öffnet. Die Zonierung des Raumes sieht vor, dass die Ruhe von Ost (Thekenberatung, Scanstationen) nach West (PC-Arbeitsplätze, Leselounge) hin zunimmt. Wie im Multimediazentrum im Obergeschoss erfolgte die Möblierung mit Mobiliar von Bibliotheks- und Büroausstattern, um bei Bedarf flexibel Ergänzungen und Änderungen vornehmen zu können. Die Mitnahme von Taschen und Jacken ist - im Unterschied zu den Regeln der Lesesaalnutzung - in beiden Funktionsbereichen erlaubt. Damit wird auch auf unterschiedliche nutzerseitige Bedarfe reagiert.

Im Sockelgeschoss, ein Gewölbe, das sich ebenerdig zum Innenhof öffnet, sind Flächen für Lernpausen entstanden. Hier sind mehrere Verkaufsautomaten des Studierendenwerks Heidelberg für Heiß- und Kaltgetränke, Snacks und kleinere Gerichte aufgestellt, ferner der Automat eines Dienstleisters für den Verkauf von durchsichtigen Bibliothekstaschen und Bürobedarf. Der Raum im Sockelgeschoss kann auch als Vortragsraum genutzt werden. Alle drei Räume verfügen über eine leistungsstarke WLANAbdeckung.

\section{Bewertung, derzeitige Lage, Ausblick}

Für die Monate Mai 2019 bis Februar 2020 ist für die Wiedereröffnung nach Sanierung ein insgesamt positives 
Feedback zu ziehen. Insbesondere die PC-Arbeitsplätze im Multimediazentrum werden sehr gut angenommen. Dies überrascht, da grundsätzlich von einer zunehmend mobilen Nutzung mit eigenen Geräten (Laptops, Tablets und Smartphones) auszugehen ist. Eine Erklärung hierfür könnte neben der schnellen Internetverbindung und den angebotenen Programmen sein, dass diese PCs gerne für die ungestörte Anfertigung von Seminararbeiten genutzt werden. Sollte sich dieser Trend abschwächen, kann durch Umwidmung in einfache Laptoparbeitsplätze schnell reagiert werden.

Im Informationszentrum ist die Neuausrichtung der Auskunftstheke positiv zu bewerten, die als zentraler Anlaufpunkt für Fragen rund um die Bibliotheksnutzung nun einladender gestaltet ist. Auch dies steht einem allgemeinen Trend zur zunehmend mobilen Auskunft entgegen, die darauf abzielt, die Nutzerinnen und Nutzer aktiv aufzusuchen. Angesichts der Größe und Nutzungsfrequenz durch sehr heterogene Nutzergruppen erscheint jedoch dieser eine zentrale Anlaufpunkt auch langfristig erforderlich zu sein. Abgesehen von der Auskunftstheke ist die Nutzung im Informationszentrum im Vergleich zum Multimediazentrum nach der Sanierung verhaltener. Dies liegt zum einen daran, dass hier die Arbeit am PC durch externe Nutzerinnen und Nutzer trotz der Reduzierung der Geräte von 46 auf 36 überproportional rückläufig ist, zum anderen weisen Nutzerkommentare darauf hin, dass aufgrund des erhabenen Charakters des Raumes erst eine Gewöhnung und Aneignung stattfinden muss. Angesichts der alternativen Nutzungsangebote, zu denen auch das informelle Arbeiten im Pausenraum im Sockelgeschoss gehört, wird dies jedoch nicht als kritisch bewertet. In Bezug auf die Anzahl der Recherche-PCs ist auch hier aufgrund der flexiblen Möblierung bei Bedarf eine Umwidmung in mit Steckdosen ausgestattete freie Nutzerarbeitsplätze leicht herzustellen.

Nach der Schließung der Bibliothek im Zuge der Corona-Maßnahmen am 18. März 2020 wurde das Informationszentrum am 28. April, das Multimediazentrum am 2. Juni unter Einschränkungen wieder geöffnet. Im Informationszentrum sind derzeit (Stand 10/2020) die Auskunft, die Kopier- und Scanmöglichkeiten, 3 PCs für Kurzrecherchen sowie 12 Recherche-PCs zugänglich. Die LeseLounge sowie die übrigen Recherche-PCs sind geschlossen. Im Multimediazentrum stehen 26 PC-Arbeitsplätze für die Buchung durch Mitglieder der Universität sowie die Sondernutzungsplätze zur Verfügung. Die freien Laptoparbeitsplätze sowie die weiteren PC-Arbeitsplätze sind derzeit nicht zugänglich. Auch hier erwiesen sich in beiden Räumen die Auskunftstheken, die während der gesamten Öffnungszeit mit Aufsicht führendem Personal besetzt sind, als unverzichtbar. Die Flexibilität der Möblierung ermöglicht die schnelle Reaktion auf ein der jeweiligen Nutzungssituation angepasstes Nutzungskonzept.

Mit der Sanierung des Südflügels der Universitätsbibliothek Heidelberg auf drei Geschossen ist ein weiterer wichtiger Meilenstein der Generalsanierung der Universitätsbibliothek erreicht. Weitere Maßnahmen, die insbesondere den Abschluss der Sanierung des Magazintrakts, des Dachgeschosses des historischen Altbaus sowie die Sanierung der Sandsteinfassade umfassen, werden in zwei Bauabschnitten 2019-24 folgen. ${ }^{11}$ Ziel ist es, dass - nach dem Abschluss der Sanierung des Triplex-Gebäudes im Jahr 2015 - dann der gesamte Altbau der Universitätsbibliothek saniert und für die Zukunft ertüchtigt sein wird.

\section{Literaturverzeichnis}

Rosenbusch, Harry (1900): Aus der Geologie von Heidelberg. Akademische Rede zur Feier des Geburtsfestes des höchstseligen Grossherzogs Karl Friedrich am 22. November 1900 [...] Universität Heidelberg. Universitäts-Buchdruckerei von J. Hörnig.

Balzuweit, Rike; Nissen, Martin (2016): An der Zukunft bauen. Die Norderweiterung der Universitätsbibliothek Heidelberg und ihre aktuellen Innovationsfelder. In: BIBLIOTHEK - Forschung und Praxis, 40 (3), 452-67.

Ewald, Gustav (1989): Planen und Bauen für die Universitätsbibliothek Heidelberg (1953-1987). In: Bibliothek im Wandel: ein Werkstattbericht über die Sanierung des Gebäudes der Universitätsbibliothek Heidelberg, hg. v. Elmar Mittler. Heidelberg: Heidelberger Verl.-Anstalt, 33-104.

Stichs, Hans Peter (1989): Zur Restaurierung und den Erweiterungsbauten der Universitätsbibliothek in den Jahren 1985-1987. In: Bibliothek im Wandel: ein Werkstattbericht über die Sanierung des Gebäudes der Universitätsbibliothek Heidelberg, hg. v. Elmar Mittler. Heidelberg: Heidelberger Verl.- Anstalt, 124-33. Probst, Veit; Balzuweit, Rike (2020): Die Zukunft des Heidelberger Bibliothekssystems im Spiegel des CATS-Forschungsbaus. In: ABI-Technik, 40, 127-38.

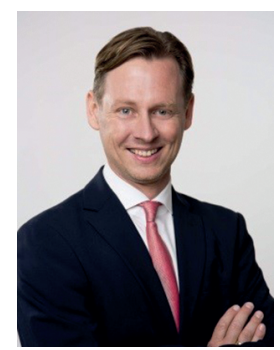

Dr. Martin Nissen

Universitätsbibliothek Heidelberg

Plöck 107-109

D-69117 Heidelberg

Nissen@ub.uni-heidelberg.de

11 Vgl. Probst und Balzuweit (2020) 136. 